\title{
Unsteady computational fluid dynamics modeling of free-flight projectile aerodynamics
}

\author{
J. Sahu \\ U.S. Army Research Laboratory, APG, MD, USA
}

\begin{abstract}
This paper describes a new multidisciplinary computational study undertaken to model the flight trajectories and the free-flight aerodynamics of both a finned projectile at supersonic velocities and a spinning projectile at subsonic speeds with and without aerodynamic flow-control. Actual flight trajectories are computed using an advanced coupled computational fluid dynamics (CFD)/rigid body dynamics (RBD) technique. An advanced time-accurate Navier-Stokes computational technique has been used in CFD to compute the unsteady aerodynamics associated with the free-flight of the finned projectile at supersonic speeds and the spinning projectile at subsonic speeds. Computed positions and orientations of the projectile have been compared with actual data measured from free-flight tests and are found to be generally in good agreement. Predicted aerodynamics forces and moments compare well with the forces and moments used in the six degree freedom fits of the results of the same tests. Unsteady numerical results obtained from the coupled method show the flow field, the aerodynamic forces and moments, and the flight trajectories of the projectile.

Keywords: unsteady CFD, projectile aerodynamics, couple CFD/RBD method, flight trajectory.
\end{abstract}

\section{Introduction}

Understanding the aerodynamics of projectiles, rockets, and missiles is critical to the design of stable configurations and contributes significantly to the overall performance of weapon systems [1-3]. The prediction of aerodynamic coefficients for these weapon systems is essential in assessing the performance of new designs. Numerical simulations have the potential of greatly reducing design costs while providing a detailed understanding of the complex 
aerodynamics associated with each change. Recently, we have made progress in coupling computational fluid dynamics and flight dynamics to perform required multidisciplinary simulations for moving body problems. This involves realtime multidisciplinary-coupled computational fluid dynamics/rigid body aerodynamics computations for the entire flight trajectory of a complex guided projectile system. It can lead to accurate determination of aerodynamics that is critical to the low-cost development of new advanced guided projectiles, rockets, missiles, and smart munitions.

Improved computer technology and state-of-the-art numerical procedures now enable solutions to complex, 3-D problems associated with projectile and missile aerodynamics. Modern guided munitions for future combat systems require the use of complex control surfaces (fins and canards), control mechanisms, and/or the use of flow technologies such as microjet gas generators to provide maneuver authority. The aerodynamic flow fields over these Army weapons are complex involving non-linear flow-physics especially during and after control maneuvers. For maneuvering munitions however, very limited data is available during and after control maneuvers [4,5], and there is a lack of knowledge and understanding of the associated unsteady aerodynamics. Accurate numerical modeling of this unsteady aerodynamics has been found to be challenging both in terms of time-accurate solution techniques and computing resources required. Our goal is to be able to perform time-accurate multidisciplinary-coupled computational fluid dynamics (CFD) and rigid body dynamics (RBD) computations for complex guided projectiles with control maneuvers using microjets and/or control surfaces such as fins/canards. As part of a DOD High Performance Computing Grand Challenge Project, the present work is focused on the coupling of CFD and rigid body dynamics (RBD) techniques for simultaneous prediction of the unsteady free-flight aerodynamics and the flight trajectory of projectiles. In other words, can we perform physics-based fly-outs of the projectiles on the supercomputers and accurately predict the unsteady aerodynamics and flight behavior of projectiles in actual flights?

\section{Technical approach}

A real-time accurate approach is used in the present work; however, timeaccurate computations require much greater computer resources. The real-time accurate approach also requires that the six-degrees-of-freedom (6 DOF) body dynamics be computed at each repetition of the fluid flow solver. In threedimensional space, a rigid object has 6 DOF: three translations and three rotations. The 6 DOF code computes linear and angular velocities as well as the orientation of the missile, which are used as input to the computational fluid dynamics code. In turn, the aerodynamic forces and moments obtained from the flow solver are used to solve the 6 DOF body dynamics before moving on to the next time step. This procedure allows one to perform real-time multidisciplinary-coupled computational fluid dynamics/rigid body aerodynamics computations for the partial or entire flight trajectory of a complex guided projectile system. 
The CFD capability used here solves the Navier-Stokes equations [6-9] and incorporates advanced boundary conditions and grid motion capabilities. The present numerical study is a big step forward and a direct extension of that research which now includes numerical simulation of the actual fight paths of the projectile using coupled $\mathrm{CFD} / \mathrm{RBD}$ techniques using real-time accurate approach. The complete set of 3-D time-dependent Navier-Stokes equations is solved in a time-accurate manner for simulations of actual flights. The basic numerical framework in the code contains unified-grid, unified-physics, and unified-computing features. The user is referred to these references for details of the basic numerical framework. The 3-D time-dependent Reynolds-averaged Navier-Stokes (RANS) equations are solved using the finite volume method [7]:

$$
\frac{\partial}{\partial t} \int_{V} \mathbf{W} d V+\oint[\mathbf{F}-\mathbf{G}] \cdot d A=\int_{V} \mathbf{H} d V
$$

where $\mathbf{W}$ is the vector of conservative variables, $\mathbf{F}$ and $\mathbf{G}$ are the inviscid and viscous flux vectors, respectively, $\mathbf{H}$ is the vector of source terms, $\mathrm{V}$ is the cell volume, and $\mathrm{A}$ is the surface area of the cell face.

Second-order discretization was used for the flow variables and the turbulent viscosity equation. The turbulence closure is based on topology-parameter-free formulations. Two-equation [6] and higher order hybrid RANS/LES [10,11] turbulence models were used for the computation of turbulent flows. The hybrid RANS/LES approach is well suited to the simulation of unsteady flows and contains no additional empirical constants beyond those appearing in the original RANS and LES sub-grid models. With this method a regular RANS-type grid is used except in isolated flow regions where denser, LES-type mesh is used to resolve critical unsteady flow features. The hybrid model transitions smoothly between an LES calculation and a cubic k- $\varepsilon$ model, depending on grid fineness. For computations of unsteady flow fields that are of interest here, dual timestepping as described below was used to achieve the desired time-accuracy [12].

An unique feature of the present coupled approach is the full grid motion capability that allows the grid to move translate and rotate as the projectile flies down the rage, since the grid velocity is assigned to each mesh point. To account for rigid body dynamics, the grid point velocities are set as if the grid is attached to the rigid body with 6 DOF. As shown schematically in Figure 1, the 6 DOF comprise of the three spatial coordinates $(x, y, z)$ and the three Euler angles, roll, pitch, and yaw $(\phi, \Phi, \Psi)$. For the rigid body dynamics, the coupling refers to the interaction between the aerodynamic forces/moments and the dynamic response of the projectile/body to these forces and moments. The forces and moments are computed every CFD time step and transferred to a 6 DOF module which computes the body's response to the forces and moments. The response is converted into translational and rotational accelerations that are integrated to obtain translational and rotational velocities and integrated once more to obtain linear position and angular orientation. From the dynamic response, the grid point locations and grid point velocities are set. Both CFD and RBD computations are performed at every time step in a fully coupled manner. 


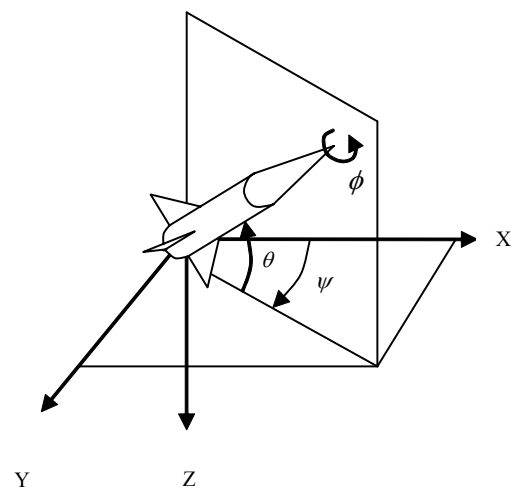

Figure 1: Rigid body dynamics schematic.

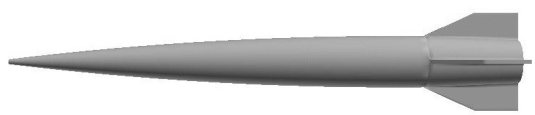

Figure 2: $\quad$ Finned configuration.

\section{Results}

Time-accurate unsteady numerical computations were performed using NavierStokes and coupled 6 DOF methods to predict the flow field and aerodynamic coefficients, and the flight paths of both a finned projectile at supersonic speeds and a spinning projectile at subsonic speeds. In all cases, full $3 \mathrm{D}$ computations were performed and no symmetry was used.

The supersonic projectile modeled in this study is an ogive-cylinder-finned configuration (see Figure 2). The length of the projectile is $121 \mathrm{~mm}$ and the diameter is $13 \mathrm{~mm}$. The ogive nose is $98.6 \mathrm{~mm}$ long and the afterbody has a $22.3 \mathrm{~mm}, 2.5^{\circ}$ boat-tail. Four fins are located on the back end of the projectile. Each fin is $22.3 \mathrm{~mm}$ long and $10.16 \mathrm{~mm}$ thick. The computational mesh for the $25-\mathrm{mm}$ projectile model is an unstructured one. In general, most of the grid points are clustered in the afterbody fin region. The total number of grid points is about 4 million for the full grid.

Here, the primary interest is in the development and application of coupled CFD and RBD techniques for accurate simulation of the free-flight aerodynamics and flight dynamics of the projectile in supersonic flight. The first step here was to obtain the steady state results for this projectile at a given initial supersonic velocity. Also imposed were the angular orientations at this stage. Corresponding converged steady state solution was then used as the starting condition along with the other initial conditions for the computation of coupled CFD/RBD runs. Numerical computations have been made for these cases at an initial velocity of $1034 \mathrm{~m} / \mathrm{s}$. The simulations were started a small distance away 
from the muzzle. The corresponding initial angle of attack was, $\alpha=4.9^{\circ}$ and initial spin rate was $2500 \mathrm{rad} / \mathrm{s}$. Fig. 3 shows the computed pressure contours at a given time or at a given location in the trajectory. It clearly shows the orientation of the body at that instant in time and the resulting asymmetric flow field due to the body at angle of attack. The orientation of the projectile of course changes from one instant in time to another as the projectile flies down range. Figure 4 shows the variation of the Euler pitch angle with distance traveled. As seen in this figure, both the amplitude and frequency in the Euler angle variation are predicted very well by the computed results and match extremely well with the data from the flight tests. One can also clearly see the amplitude damped out as the projectile flies down range i.e. with the increasing $\mathrm{x}$-distance. Although not shown here, similar behavior is observed with the Euler yaw angle and it damps out with the increasing $\mathrm{x}$-distance. The computed results again compare very well with the measured data from the flight tests.

The time histories of the pitch and yaw angles are often customarily presented as a motion plot where the pitch angle is plotted versus the yaw angle during the flight of the projectile. It represents the path traversed by the nose of the projectile during the flight trajectory (looking forward from the back of the projectile). Such a plot is shown in Figure 5. This figure shows the comparison of the motion plots obtained both from the numerical simulations and the $6 \mathrm{DOF}$ analysis of the flight results from ARFDAS [13]. Computed results match very well with the experimental flight test results.

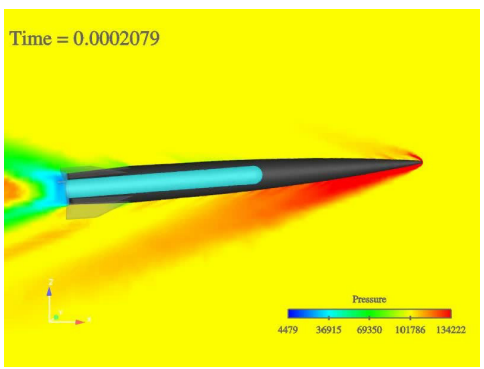

Figure 3: Computed pressure contours.

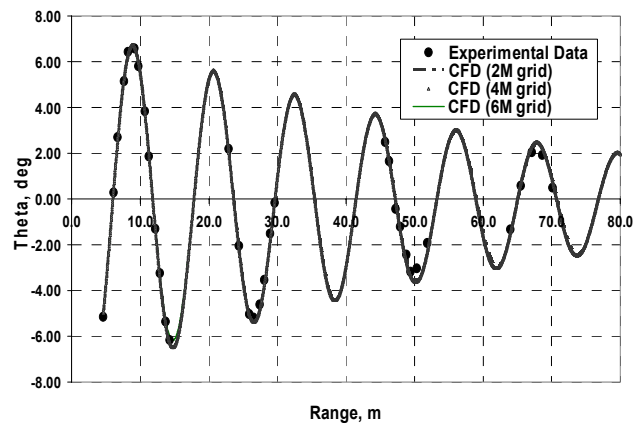

Figure 4: Euler pitch angle vs. range.

As stated earlier, coupled CFD/RBD simulations have been performed for a spinning projectile at subsonic speeds as well. The subsonic projectile is a 1.8caliber ogive-cylinder configuration (see Figure 6). A computational grid expanded near the vicinity of the projectile is shown in Figure 7. Grid points are clustered near the jet as well as the boundary layer regions to capture the high gradients flow regions. The computational grid has 211 points in the streamwise direction, 241 in the circumferential direction, and 80 in the normal direction. The unsteady simulations took thousands of hours of CPU time on the Xeon PC cluster system at ARL MSRC running with either 32 to 64 processors. 


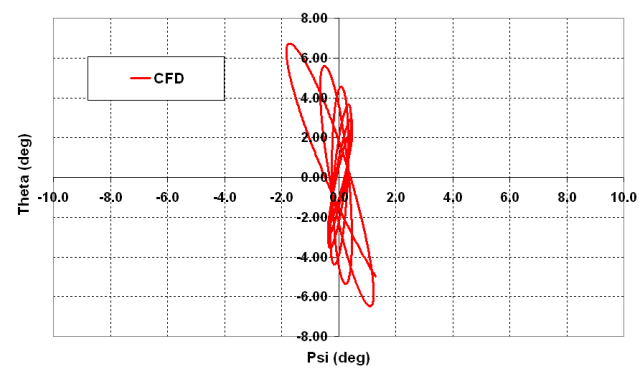

(a)

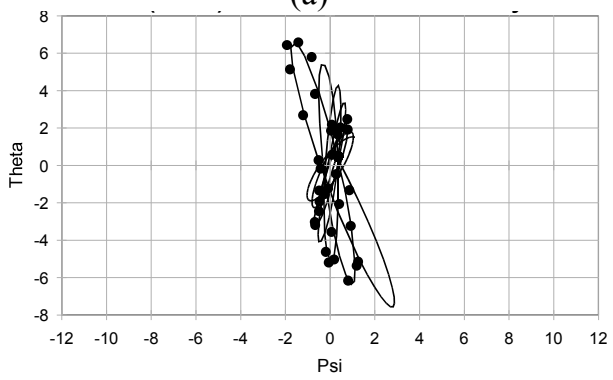

(b)

Figure 5: $\quad$ Motion plot (a) computation, (b) flight test.

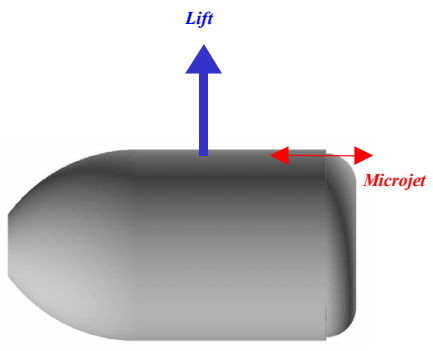

Figure 6: Spinning projectile geometry.

The first step here was to obtain the steady state results for the same projectile without the jet with the grid velocities prescribed. Also imposed were the angular orientations at this stage. Corresponding converged jet-off steady state solution was then used as the starting condition along with the other initial conditions for the computation of coupled CFD/RBD runs. Synthetic jets were activated at a specified point in the trajectory. The jet locations on the projectile are shown in Figure 6. The jet conditions were specified at the exit of the jet (sinusoidal variation in jet velocity). The jet conditions specified include the jet pressure, density and velocity components. The flow field inside of the tiny jet cavity is not computed. For the unsteady jets, time-dependent jet boundary conditions are applied at the jet exit. Numerical computations have been made for these jet cases at an initial Mach number, $M=0.39$, initial angle of attack, $\alpha$ 
$=2^{\circ}$, and an initial spin rate of $434 \mathrm{~Hz}$. The jet width was $0.32 \mathrm{~mm}$, the jet slot half-angle was $18^{\circ}$, and the peak jet velocity used was $110 \mathrm{~m} / \mathrm{s}$ operating at a frequency of $1000 \mathrm{~Hz}$. The jet-off coupled calculations are started from the same initial conditions.

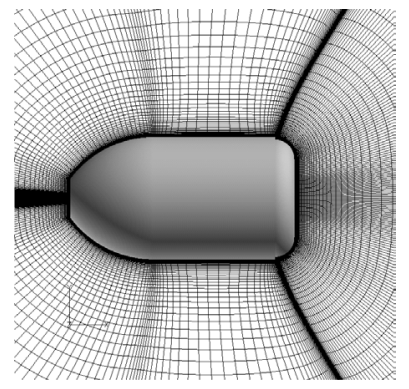

Figure 7: $\quad$ Computational grid. $\quad$ Figure 8:

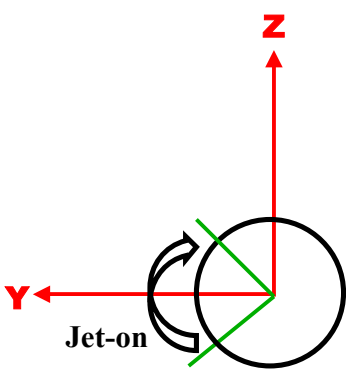

Schematic showing the jet actuation in a spin cycle).

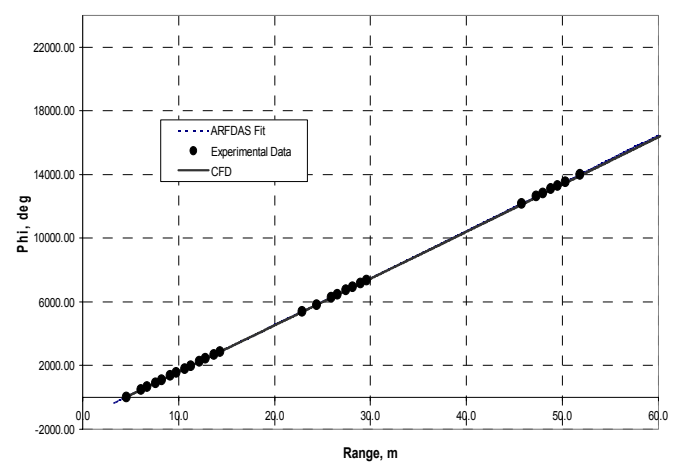

Figure 9: Comparison of computed roll angle, jet-off.

Unsteady time-accurate CFD computations require huge computer resources. All the results presented here were obtained from unsteady numerical computations with a single synthetic jet on a $40-\mathrm{mm}$ subsonic grenade (Figure 6). Looking from the back of the projectile (see Figure 8), the projectile spins clockwise. The jet actuation corresponds to one-fourth of the spin cycle from $45^{\circ}$ to $+45^{\circ}$ with zero degree being the positive y-axis. The jet is off during the remaining three-fourths of the spin cycle. The unsteady CFD modeling technique required about 180 time steps to resolve a full spin cycle. The unsteady synthetic jet operates at a high frequency of $1000 \mathrm{~Hz}$. For the part of the spin cycle when the jet is on, the jet operated for approximately four cycles. Trajectories were computed for both jet-off and jet-on conditions. For the jet-on case $^{12}$, an unsteady synthetic jet was applied while the projectile is spinning and the jet was activated at approximately 41 meters into the trajectory. Figure 9 
shows the computed roll angles as a function of the $\mathrm{x}$-distance or the range. The roll angles shown here are the accumulated values to include 360 degrees for every spin cycle of the projectile. Computed roll angle are compared with the experimentally observed values as well as data obtained the 6 DOF analysis of the flight results from ARFDAS [13]. and are found to be in good agreement.

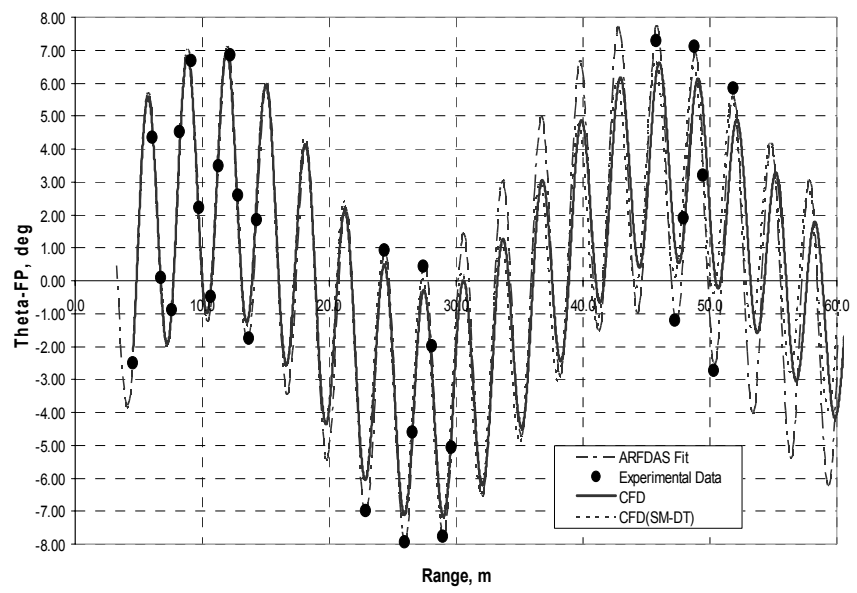

Figure 10: Computed Euler pitch angle, jet-off for different time steps.

Figure 10 shows the variation of the Euler pitch angle with distance traveled. As seen in this figure, both the amplitude and frequency in the Euler pitch angle variation are predicted very well by the computed results and match well with the data from the flight tests for up to a distance of 20 meters. For the rest of the trajectory, the frequency of the motion is predicted very well as seen by the comparison with the data, but the amplitude doesn't compare as well with the data. Two sets of CFD results are shown in this figure, one obtained with the original time step and another one with a smaller time step reduced by half. As seen in this figure, reducing time-step by half has negligible effect on the accuracy of the computed results. Further analysis and investigation are required and are underway. Figure 11 shows similar behavior with Euler yaw angle with $\mathrm{x}$-distance. The agreement between the computed results and the experimental data again is generally good except for the amplitude comparison at greater ranges. In both figures 10 and 11, we have both slow and fast mode frequencies and both compare well with the data and the ARFDAS fits. Figure 12 shows the variation of the Euler pitch angle with distance traveled both for jet-off and jeton conditions. As seen in this figure, the effect of the jet is small and incremental. The computed Euler angles are again compared with the experimental range test data shown in dark circles for the jet-off case. As seen here, the computed data predicts the frequency rather well; however, the amplitudes in some parts are under-predicted in the computations. 


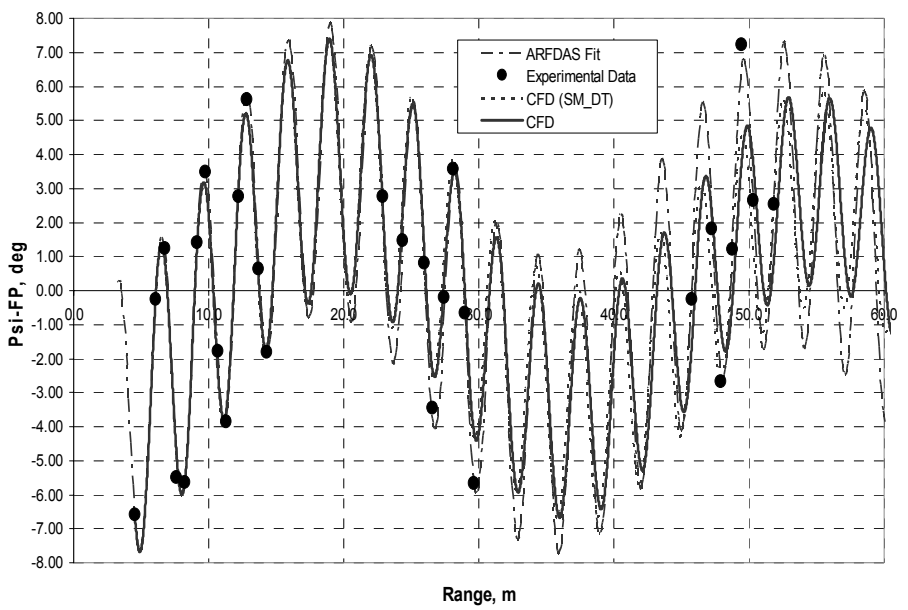

Figure 11: Comparison of the computed Euler yaw angle, jet-off for different time steps.

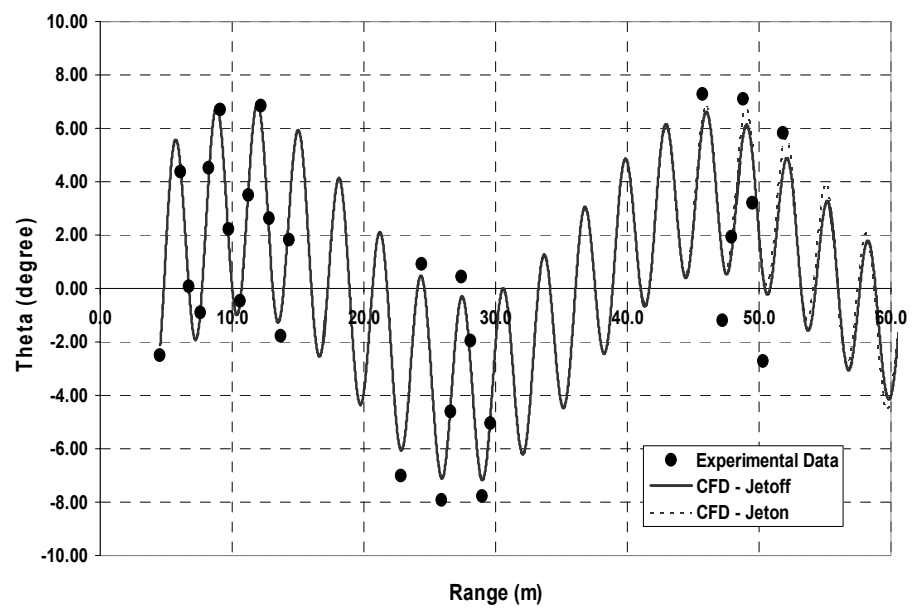

Figure 12: Comparison of computed Euler pitch angle, jet-off and jet-on.

\section{Concluding remarks}

This paper describes a new coupled CFD/RBD computational study undertaken to determine the flight aerodynamics of both a finned projectile and a spinning projectile. A three-dimensional unsteady Navier-Stokes solver is employed to compute the time-accurate aerodynamics associated with the free-flight of the finned projectile at supersonic velocities and the spinning projectile at subsonic speeds. Computed positions and orientations of the projectile have been compared with actual data measured from free-flight tests and are found to be generally in good agreement. Predicted aerodynamics forces and moments also compare well with the forces and moments used in the six degree freedom fits of 
the results of the same tests. This work demonstrates a coupled method to accurately predict the time-accurate unsteady aerodynamics and the flight trajectories of projectiles at various speeds. The present CFD/RBD simulations clearly show the capability of the coupled approach and form the basis for future multidisciplinary, time-dependent computations of advanced maneuvering munitions.

\section{References}

[1] Sahu, J., K. R. Heavey, and E. N. Ferry, "Computational Fluid Dynamics for Multiple Projectile Configurations." Proceedings of the $3^{\text {rd }}$ Overset Composite Grid and Solution Technology Symposium, Los Alamos, NM, October 1996.

[2] Sahu, J., K. R. Heavey, and C. J. Nietubicz, "Time-Dependent NavierStokes Computations for Submunitions in Relative Motion." $6^{\text {th }}$ International Symposium on Computational Fluid Dynamics, Lake Tahoe, NV, September 1995.

[3] Meakin, R. L., "Computations of the Unsteady Flow about a Generic Wing/Pylon/Finned-Store Configuration.” AIAA 92-4568-CP, August 1992.

[4] Smith B. L., and A. Glezer, "The Formation and Evolution of Synthetic Jets.” Journal of Physics of Fluids, vol. 10, No. 9, September 1998.

[5] Davis, S. A. and Glezer, A., "The Manipulation of Large- and SmallScales in Coaxial Jets using Synthetic Jet Actuators", AIAA Paper No. 2000-0403, January 2000.

[6] Avancha, R. and Pletcher, R. H., "Large Eddy Simulation of the Turbulent Flow Past a Backward Facing Step", AIAA paper No. 2000-0542, January 2000.

[7] Arunajatesan, S. and Sinha, N., "Towards Hybrid LES-RANS Computations of Cavity Flowfields", AIAA Paper No. 2000-0401, January 2000.

[8] Batten, P., U. Goldberg and S. Chakravarthy, "Sub-grid Turbulence Modeling for Unsteady Flow with Acoustic Resonance", AIAA Paper 000473, 38th AIAA Aerospace Sciences Meeting, Reno, NV, January 2000.

[9] Peroomian, O., S. Chakravarthy, and U. Goldberg, "A 'Grid-Transparent' Methodology for CFD.” AIAA Paper 97-07245, 1997.

[10] Peroomian, O., S. Chakravarthy, S. Palaniswamy, and U. Goldberg, "Convergence Acceleration for Unified-Grid Formulation Using Preconditioned Implicit Relaxation.” AIAA Paper 98-0116, 1998.

[11] Goldberg, U. C., O. Peroomian, and S. Chakravarthy, "A Wall-DistanceFree K-E Model with Enhanced Near-Wall Treatment.” ASME Journal of Fluids Engineering, Vol. 120, pp. 457-462, 1998.

[12] Sahu, J., "Unsteady CFD Modeling of Aerodynamic Flow Control over a Spinning Body with Synthetic Jet." AIAA Paper 2004-0747, Reno, NV, 58 January 2004.

[13] Arrow Tech Associates. "ARFDAS Technical Manual." South Burlington, VT, 2001. 